SHORT REPORT

\title{
Notch3 gene polymorphism and ischaemic cerebrovascular disease
}

\author{
D Ito, N Tanahashi, M Murata, H Sato, I Saito, K Watanabe, Y Fukuuchi
}

J Neurol Neurosurg Psychiatry 2002;72:382-384

Cerebral autosomal dominant arteriopathy with subcortical infarcts and leukoencephalopathy (CADASIL) is a type of hereditary stroke and dementia. More than $90 \%$ of patients with CADASIL have mutations in the Notch3 gene. All mutations either create or destroy a cysteine residue in the epidermal growth factor-like repeats. In addition, five polymorphisms, which lead to amino acid substitutions, have been identified within the Notch3 coding sequence. However, whether these polymorphisms affect Notch signalling or are involved in cerebrovascular diseases is unknown. In the present study, we investigated a possible association between a T6746C polymorphism in the Notch3 coding region and the occurrence of symptomatic ischaemic cerebrovascular disease (CVD) was investigated. Two hundred and thirty five patients with CVD, as confirmed by brain CT or MRI, and 315 age and sex matched control subjects were analyzed for genotype frequencies of the T6746C polymorphism in Notch3. The genotype distributions were: patients with CVD, C/C $14.0 \%, C / T 45.5 \%$, and $\mathrm{T} / \mathrm{T} 40.4 \%$; controls, $\mathrm{C} / \mathrm{C}$, $14.3 \% ; C / T, 47.9 \% ; T / T, 37.8 \%$. The Japanese population has a higher $\mathrm{C}$ allele frequency of the T6746C polymorphism than European populations. There was no significant difference between the T6746C polymorphism in patients with CVD and controls $\left(\chi^{2}=0.414, p=0.813\right)$. This was confirmed by the results of multiple logistic regression analysis including established risk factors $\left(\chi^{2}\right.$ $=4.65, p=0.311)$. In conclusion, the results indicate that T6746C polymorphism in the intracellular domain of the Notch3 gene is not associated with an increased risk for CVD.

C erebral autosomal dominant arteriopathy with subcortical infarcts and leukoencephalopathy (CADASIL) is a type of hereditary stroke and dementia, key features of which include recurrent subcortical ischaemic events and vascular dementia associated with diffuse white matter abnormalities on neuroimaging. This disease has now been reported from many regions worldwide. In 1994, CADASIL was reportedly linked to chromosome 19 and positional cloning led to the identification of mutations in the Notch3 gene in $1996 .{ }^{1}$ This gene encodes a 2321 amino acid type I transmembrane protein that is thought to be involved in cell signalling and fate during embryonic development. The extracellular ligand binding domain contains 34 tandemly repeated epidermal growth factor (EGF)-like repeats, and the intracellular domain is thought to be involved in signal transduction. ${ }^{2}$

Missense mutations in Notch3 were found in $90 \%$ of patients with CADASIL. Joutel et al have systematically screened for mutations in the 33 coding exons of the Notch 3 gene in unrelated patients and have identified 25 point mutations associated with this disease, as well as a clustering of mutations within exons 3 and $4 .^{3}$ All mutations either create

$\begin{aligned} & \text { Table } 1 \text { Clinical characteristics of patients with CVD } \\
& \text { and controls }\end{aligned}$
\begin{tabular}{llll} 
& & \\
\hline & $\begin{array}{l}\text { Controls } \\
(\mathrm{n}=315)\end{array}$ & $\begin{array}{l}\text { CVD patients } \\
(\mathrm{n}=235)\end{array}$ & p Value* \\
\hline Male (\%) & 76.2 & 77.9 & $\mathrm{NS}$ \\
Age (y, mean (SD)) & $58.6(4.3)$ & $58.3 \pm 7.8$ & $\mathrm{NS}$ \\
Family history (\%) & 23.2 & 29.3 & 0.200 \\
Hypertension (\%) & 24.7 & 56.2 & $<0.001$ \\
Hypercholesterolaemia (\%) & 32.4 & 38.6 & 0.130 \\
Diabetes mellitus (\%) & 6.3 & 25.8 & $<0.001$ \\
Smoking (\%) & 37.5 & 53.3 & $<0.001$ \\
\hline
\end{tabular}

${ }^{*} \chi^{2}$ tests were used to compare the values of patients with CVD and controls for all parameters except age, which was compared using Student's $t$ test.

or destroy a cysteine residue in the EGF-like repeats. In addition, they also identified, within the Notch3 coding sequence, 15 polymorphisms in an unaffected group. Five of these polymorphisms lead to amino acid substitutions in the coding region. It is unknown, however, whether these polymorphisms affect Notch signalling or are involved in cerebrovascular diseases. The aim of this study was to determine whether the most common polymorphism, T6746C, in the intracellular domain of the Notch3 gene is associated with symptomatic ischaemic cerebrovascular disease (CVD).

\section{METHODS \\ Subjects}

We analyzed 235 unrelated Japanese patients with CVD and 315 age and sex matched control subjects. All patients had visited the outpatient clinic of Keio University Hospital in Tokyo for regular follow up examinations. We selected patients aged 70 years or less at the onset of CVD. On the basis of the classification of cerebrovascular diseases III report from the committee established by the National Institute of Neurological Disorders and Stroke, patients diagnosed with atherothrombotic infarction, lacunar infarction, or transient ischaemic attack (TIA) were enrolled in this study. Those with cardioembolic cerebral infarction and cerebral haemorrhage were excluded. Controls were patients who had had regular check ups. Those with a clinical history of cerebrovascular disease, myocardial infarction, or peripheral vascular disease were excluded. Written informed consent was obtained from all subjects after a full explanation of the study and a guarantee of total privacy. Brain CT and/or MRI was performed on all patients with CVD. Hypertension, hypercholesterolaemia, and smokers were defined as described previously. ${ }^{4-6}$

Abbreviations: CADASIL, Cerebral autosomal dominant arteriopathy with subcortical infarcts and leukoencephalopathy; CVD, cerebrovascular disease; EGF, epidermal growth factor; TIA, transient ischaemic attack; $\mathrm{PCR}$, polymerase chain reaction 
Table 2 Allele and genotype frequencies of the Notch3 polymorphism in patients with CVD and controls

\begin{tabular}{|c|c|c|c|c|c|c|c|}
\hline & \multicolumn{4}{|c|}{ Genotype (\%) } & \multicolumn{3}{|c|}{ Allele frequency (\%) } \\
\hline & $\mathrm{CC}$ & CT & TT & p Value* & $\mathrm{C}$ & $\mathrm{T}$ & $\mathrm{p}$ Value \\
\hline Controls ( $n=315$ ) & 14.3 & 47.9 & 37.8 & & 38.3 & 61.7 & \\
\hline All CVD patients ( $n=235$ ) & 14.0 & 45.5 & 40.4 & 0.813 & 36.8 & 63.2 & 0.625 \\
\hline Atherothrombotic $(n=69)$ & 15.9 & 55.1 & 29.0 & 0.387 & 43.5 & 56.5 & 0.248 \\
\hline Lacunar $(n=142)$ & 14.1 & 38.7 & 47.2 & 0.137 & 33.5 & 66.5 & 0.171 \\
\hline TIA $(n=24)$ & 8.3 & 58.3 & 33.3 & 0.554 & 37.5 & 62.5 & 0.917 \\
\hline
\end{tabular}

${ }^{*} \chi^{2}$ tests were used to compare genotypes between controls and all patients with CVD, and between controls and subgroups of patients with CVD; tallele frequencies were compared using Student's $t$ test. TIA, transient ischaemic attack

\section{Polymorphism analysis}

To analyze the T6746C polymorphism of Notch3, the polymerase chain reaction (PCR) was carried out as described previously. ${ }^{4-6}$ Amplification of a 203 bp fragment of the Notch3 gene was performed with the $5^{\prime}$ primer $5^{\prime}$-CTTACCTGG CAGTCCCAGG-3' and $3^{\prime}$ primer 5'-AGTGGCAGTGGCT GGGCTAG-3'. The PCR consisted of 1 cycle of 15 minutes at $80^{\circ} \mathrm{C}$ and 4.5 minutes at $94^{\circ} \mathrm{C}, 43$ cycles of 1 minute at $94^{\circ} \mathrm{C}, 1$ minute at $65^{\circ} \mathrm{C}$, and 45 seconds at $72^{\circ} \mathrm{C}$, followed by 7 minutes at $72^{\circ} \mathrm{C}$ in a Gene Amp PCR system 2400 (Perkin Elmer, Foster City, CA, USA). The PCR product $(6 \mu \mathrm{l})$ was cleaved with $0.5 \mathrm{U}$ of Mwo I restriction enzyme (New England Biolabs, Beverly, MA, USA). Digestion of the PCR products yielded bands of 203 bp in TT homozygotes, 158 bp in CC homozygotes, and both bands in heterozygotes.

\section{Statistical analysis}

The differences in genotype frequencies and other risk factors were analyzed by the $\chi^{2}$ test. Mean age in the two groups and the allele frequency were compared by Student's $t$ test. Multiple logistic regression methods were used to control for possible confounding factors. All statistical analyses were performed using Statview software (ver 5.0 for windows, SAS Institute, CA, USA).

\section{RESULTS}

Table 1 summarises the clinical features of the patients with CVD and the control subjects studied. There were no significant differences in age or sex between the two groups. The risk factors hypertension, diabetes mellitus, and smoking were significantly more common in the patients with CVD. Although the frequency of a family history of stroke was higher in patients with CVD, the difference between the patients with CVD and controls was not statistically significant.

The genotype distributions and allelic frequencies of the T6746C polymorphism in Notch3 in the control and CVD groups are shown in table 2. Among the patients with CVD, $14.0 \%$ were $\mathrm{C} / \mathrm{C}, 45.5 \%$ were $\mathrm{C} / \mathrm{T}$, and $40.4 \%$ were $\mathrm{T} / \mathrm{T}$. This genotype distribution was not significantly different from the distribution in the control group (C/C, $14.3 \%$; $\mathrm{C} / \mathrm{T}, 47.9 \%$; $\mathrm{T} / \mathrm{T}$, $37.8 \%)\left(\chi^{2}=0.414, p=0.813\right)$. C allele frequencies were also similar in patients with CVD and controls $(36.8 \%$ and $38.3 \%$, respectively). This was confirmed by the results of multiple logistic regression analysis with the established risk factors, family history, hypertension, hypercholesterolaemia, diabetes mellitus, and smoking $\left(\chi^{2}=4.65, p=0.311\right)$. In further calculations, low risk subgroups (excluding those with family history, hypertension, hypercholesterolaemia, diabetes mellitus, or smoking) were analyzed. The Notch3 genotype was not related to CVD in any of the subgroups (data not shown). In analyzing subjects with a positive family history, there was no difference in genotype between the CVD and control groups.
As shown in table 2, analysis by CVD subtype (atherothrombotic infarction, lacunar infarction, TIA) also failed to yield any difference in genotype distribution compared with the control group.

\section{DISCUSSION}

The present study is the first to examine the relation between CVD and the Notch3 polymorphism. The Japanese population has a higher $\mathrm{C}$ allele frequency in T6746C polymorphism than European populations. ${ }^{3}$ Our study shows that the Notch3 polymorphism is not associated with CVD, even in low risk subjects.

Mutations in the Notch3 gene are missense mutations characteristically leading to the loss or gain of a cysteine residue in one of the EGF-like domains of the protein. ${ }^{3}$ The abnormal Notch3 allele may encode for a protein product with an abnormal conformation due to disruption of the disulphide bonding of cysteine residues. Five genetic polymorphisms, which lead to amino acid substitutions, have been reported in the coding sequence of Notch 3. ${ }^{3}$ Among them, the most common polymorphism, T6746C, results in an amino acid dimorphism (Val/Ala) at residue 2223, which is located in the intracellular domain. Because the intracellular domain of Notch3 is thought to be involved in signal transduction, ${ }^{2}$ this polymorphism has been suggested to be directly associated with Notch3 function. However, our data yielded no evidence of an association between T6746C polymorphism and CVD. In our preliminary study, we analyzed other polymorphisms in the Notch3 coding region, although they seem to be rare in the Japanese population. Therefore, it is unlikely that they are associated with common diseases, such as CVD.

Finally, several points should be kept in mind when interpreting the results of the present study. Our study refers to the association between this polymorphism and CVD only in the Japanese population, where the prevalence of stroke is especially higher than that in most western European countries. The relevance of this polymorphism should be investigated in other populations as well as in prospective studies. Moreover, many patients with CVD in this study also had leukoencephalopathy, which is a key feature of CADASIL on neuroimaging. Therefore, it is necessary to examine the relation between white matter lesions and Notch3 polymorphism. Although the present study failed to find a relation between Notch3 polymorphism and CVD, further studies are necessary to evaluate whether Notch3 may play a part in CVD.

\section{ACKNOWLEDGEMENTS}

This study was supported by the Kanae Foundation for Life and Socio-Medical Science. No competing interests declared. 


\section{Authors' affiliations}

D Ito, N Tanahashi, H Sato, Y Fukuuchi, Department of Neurology, School of Medicine, Keio University, Tokyo, Japan

M Murata, K Watanabe, Department of Laboratory Medicine, School of Medicine, Keio University, Tokyo, Japan

M Murata, Department of Hematology, School of Medicine, Keio

University, Tokyo, Japan

I Saito, Health Center, School of Medicine, Keio University, Tokyo, Japan

Correspondence to: Dr D lto, Department of Neurology, School of Medicine, Keio University, 35 Shinanomachi, Shinjuku-ku, Tokyo 160-8582, Japan; di49@med.keio.ac.jp

Received 11 June 2001

In revised form 1 November 2001

Accepted 8 November 2001

\section{REFERENCES}

1 Joutel A, Corpechot C, Ducros A, et al. Notch 3 mutations in CADASIL, a hereditary adult-onset condition causing stroke and dementia. Nature 1996:383:707-10

2 Weinmaster $\mathbf{G}$. The ins and outs of notch signaling. Mol Cell Neurosci 1997;9:91-102.

3 Joutel A, Vahedi K, Corpechot C, et al. Strong clustering and stereotyped nature of Notch3 mutations in CADASIL patients. Lancet 1997;350:1511-15

4 Sonoda A, Murata $M$, Ito $D$, et al. Association between platelet glycoprotein lbá genotype and ischemic cerebrovascular disease. Stroke 2000;31:493-7.

5 Ito $\mathrm{D}$, Murata M, Watanabe K, et al. C242T polymorphism of NADPH oxidase p22 PHOX gene and ischemic cerebrovascular disease in the Japanese population. Stroke 2000;31:936-9.

6 Ito D, Murata M, Tanahashi N, et al. Polymorphism in the promoter of lipopolysaccharide receptor CD14 and ischemic cerebrovascular disease. Stroke 2000;31:2661-4.

\section{Readers' favourite}

\section{Top 10}

Click on the "Top 10" button on the homepage

to see which are the best read articles each month

www.jnnp.com 